\title{
Newton numbers and residual measures of plurisubharmonic functions
}

\author{
by Alexander Rashkovskit (Kharkov)
}

\begin{abstract}
We study the masses charged by $\left(d d^{c} u\right)^{n}$ at isolated singularity points of plurisubharmonic functions $u$. This is done by means of the local indicators of plurisubharmonic functions introduced in [15]. As a consequence, bounds for the masses are obtained in terms of the directional Lelong numbers of $u$, and the notion of the Newton number for a holomorphic mapping is extended to arbitrary plurisubharmonic functions. We also describe the local indicator of $u$ as the logarithmic tangent to $u$.
\end{abstract}

1. Introduction. The principal information on local behaviour of a subharmonic function $u$ in the complex plane can be obtained by studying its Riesz measure $\mu_{u}$. If $u$ has a logarithmic singularity at a point $x$, the main term of its asymptotics near $x$ is $\mu_{u}(\{x\}) \log |z-x|$. For plurisubharmonic functions $u$ in $\mathbb{C}^{n}, n>1$, the situation is not so simple. The local properties of $u$ are controlled by the current $d d^{c} u$ (we use the notation $d=\partial+\bar{\partial}, d^{c}=$ $(\partial-\bar{\partial}) /(2 \pi i))$ which cannot charge isolated points. The trace measure $\sigma_{u}=$ $d d^{c} u \wedge \beta_{n-1}$ of this current is precisely the Riesz measure of $u$; here $\beta_{p}=$ $(p !)^{-1}(\pi / 2)^{p}\left(d d^{c}|z|^{2}\right)^{p}$ is the volume element of $\mathbb{C}^{p}$. A significant role is played by the Lelong numbers $\nu(u, x)$ of the function $u$ at points $x$ :

$$
\nu(u, x)=\lim _{r \rightarrow 0}\left(\tau_{2 n-2} r^{2 n-2}\right)^{-1} \sigma_{u}\left[B^{2 n}(x, r)\right],
$$

where $\tau_{2 p}$ is the volume of the unit ball $B^{2 p}(0,1)$ of $\mathbb{C}^{p}$. If $\nu(u, x)>0$ then $\nu(u, x) \log |z-x|$ gives an upper bound for $u(z)$ near $x$; however, the difference between these two functions can be comparable to $\log |z-x|$.

Another important object generated by the current $d d^{c} u$ is the MongeAmpère measure $\left(d d^{c} u\right)^{n}$. For the definition and basic facts on the complex Monge-Ampère operator $\left(d d^{c}\right)^{n}$ and Lelong numbers, we refer the reader to the books [12], [14] and [8], and for more advanced results, to [2]. Here we

2000 Mathematics Subject Classification: 32U05, 32U25, 32W20.

Key words and phrases: plurisubharmonic function, directional Lelong number, local indicator, Monge-Ampère operator, Newton polyhedron. 
mention that $\left(d d^{c} u\right)^{n}$ cannot be defined for all plurisubharmonic functions $u$, but if $u \in \operatorname{PSH}(\Omega) \cap L_{\text {loc }}^{\infty}(\Omega \backslash K)$ with $K \subset \subset \Omega$, then $\left(d d^{c} u\right)^{n}$ is well defined as a positive closed current of bidimension $(0,0)$ (or, which is the same, as a positive measure) on $\Omega$. This measure cannot charge pluripolar subsets of $\Omega \backslash K$, and it can have positive masses at points of $K$, e.g. $\left(d d^{c} \log |z|\right)^{n}=$ $\delta(0)$, the Dirac measure at $0,|z|=\left(\sum\left|z_{j}\right|^{2}\right)^{1 / 2}$. More generally, if $f: \Omega \rightarrow$ $\mathbb{C}^{N}, N \geq n$, is a holomorphic mapping with isolated zeros at $x^{(k)} \in \Omega$ of multiplicities $m_{k}$, then $\left.\left(d d^{c} \log |f|\right)^{n}\right|_{x^{(k)}}=m_{k} \delta\left(x^{(k)}\right)$. So, the masses of $\left(d d^{c} u\right)^{n}$ at isolated singularity points of $u$ (the residual measures of $u$ ) are of especial importance.

Let a plurisubharmonic function $u$ belong to $L_{\mathrm{loc}}^{\infty}(\Omega \backslash\{x\})$; its residual mass at the point $x$ will be denoted by $\tau(u, x)$ :

$$
\tau(u, x)=\left.\left(d d^{c} u\right)^{n}\right|_{\{x\}} .
$$

The problem under consideration is to estimate this value.

The following well known relation compares $\tau(u, x)$ with the Lelong number $\nu(u, x)$ :

$$
\tau(u, x) \geq[\nu(u, x)]^{n} .
$$

Equality in (1) means that, roughly speaking, the function $u(z)$ behaves near $x$ as $\nu(u, x) \log |z-x|$. In many cases however relation (1) is not optimal; e.g. for

$$
u(z)=\sup \left\{\log \left|z_{1}\right|^{k_{1}}, \log \left|z_{2}\right|^{k_{2}}\right\}, \quad k_{1}>k_{2},
$$

we have $\tau(u, 0)=k_{1} k_{2}>k_{2}^{2}=[\nu(u, 0)]^{2}$.

As follows from the Comparison Theorem due to Demailly (see Theorem A below), the residual mass is determined by asymptotic behaviour of the function near its singularity, so one needs to find appropriate characteristics for the behaviour. To this end, a notion of local indicator was proposed in [15]. Note that $\nu(u, x)$ can be calculated as

$$
\nu(u, x)=\lim _{r \rightarrow-\infty} r^{-1} \sup \left\{v(z):|z-x| \leq e^{r}\right\}=\lim _{r \rightarrow-\infty} r^{-1} \mathcal{M}(u, x, r),
$$

where $\mathcal{M}(u, x, r)$ is the mean value of $u$ over the sphere $|z-x|=e^{r}$ (see [4]). In [5], the refined, or directional, Lelong numbers were introduced as

$$
\begin{aligned}
\nu(u, x, a) & =\lim _{r \rightarrow-\infty} r^{-1} \sup \left\{v(z):\left|z_{k}-x_{k}\right| \leq e^{r a_{k}}, 1 \leq k \leq n\right\} \\
& =\lim _{r \rightarrow-\infty} r^{-1} g(u, x, r a),
\end{aligned}
$$

where $a=\left(a_{1}, \ldots, a_{n}\right) \in \mathbb{R}_{+}^{n}$ and $g(u, x, b)$ is the mean value of $u$ over the set $\left\{z:\left|z_{k}-x_{k}\right|=\exp b_{k}, 1 \leq k \leq n\right\}$. For $x$ fixed, the collection $\{\nu(u, x, a)\}_{a \in \mathbb{R}_{+}^{n}}$ gives a more detailed information about the function $u$ near $x$ than $\nu(u, x)$ does, so one can expect a more precise bound for $\tau(u, x)$ in terms of the directional Lelong numbers. It was noticed already in [5] 
that the mean value of $u$ over $\left\{z:\left|z_{k}-x_{k}\right|=\left|\exp y_{k}\right|, 1 \leq k \leq n\right\}$ is a plurisubharmonic function of $y \in \mathbb{C}^{n}$, Re $y_{k} \ll 0$, so $a \mapsto \nu(u, x, a)$ is a concave function on $\mathbb{R}_{+}^{n}$. The idea was developed in [15] where a local indicator $\Psi_{u, x}$ of the function $u$ at $x$ was constructed as a plurisubharmonic function in the unit polydisk $D=\left\{y \in \mathbb{C}^{n}:\left|y_{k}\right|<1,1 \leq k \leq n\right\}$, given by the formula

$$
\Psi_{u, x}(y)=-\nu\left(u, x,\left(-\log \left|y_{k}\right|\right)\right)
$$

It is the largest negative plurisubharmonic function in $D$ whose directional Lelong numbers at 0 coincide with those of $u$ at $x,\left(d d^{c} \Psi_{u, x}\right)^{n}=$ $\tau\left(\Psi_{u, x}, 0\right) \delta(0)$, and finally,

$$
\tau(u, x) \geq \tau\left(\Psi_{u, x}, 0\right),
$$

so the singularity of $u$ at $x$ is controlled by its indicator $\Psi_{u, x}$.

Since $\tau\left(\Psi_{u, x}, 0\right) \geq\left[\nu\left(\Psi_{u, x}, 0\right)\right]^{n}=[\nu(u, x)]^{n},(4)$ is a refinement of (1). For the function $u$ defined by $(2), \tau\left(\Psi_{u, 0}, 0\right)=k_{1} k_{2}=\tau(u, 0)>[\nu(u, 0)]^{2}$.

Being a function of quite a simple nature, the indicator can produce effective bounds for residual measures of plurisubharmonic functions. In Theorems $1-3$ of the present paper we study the values $N(u, x):=\tau\left(\Psi_{u, x}, 0\right)$, the Newton numbers of $u$ at $x$; the reason for this name is explained below. We obtain, in particular, the following bound for $\tau(u, x)$ (Theorem 4 ):

$$
\tau(u, x) \geq \frac{[\nu(u, x, a)]^{n}}{a_{1} \ldots a_{n}} \quad \forall a \in \mathbb{R}_{+}^{n}
$$

it reduces to (1) when $a_{1}=\ldots=a_{n}=1$. For $n$ plurisubharmonic functions $u_{1}, \ldots, u_{n}$ in general position (see the definition below), we estimate the measure $d d^{c} \Psi_{u_{1}, x} \wedge \ldots \wedge d d^{c} \Psi_{u_{n}, x}$ and prove a similar relation (Theorem 6 ):

$$
\left.d d^{c} u_{1} \wedge \ldots \wedge d d^{c} u_{n}\right|_{\{x\}} \geq \frac{\prod_{j} \nu\left(u_{j}, x, a\right)}{a_{1} \ldots a_{n}} \quad \forall a \in \mathbb{R}_{+}^{n} .
$$

The main tool used to obtain these bounds is the Comparison Theorem due to Demailly. To formulate it we give the following

Definition 1. A $q$-tuple of plurisubharmonic functions $u_{1}, \ldots, u_{q}$ is said to be in general position if their unboundedness loci $A_{1}, \ldots, A_{q}$ satisfy the following condition: for all choices of indices $j_{1}<\ldots<j_{k}, k \leq q$, the $(2 q-2 k+1)$-dimensional Hausdorff measure of $A_{j_{1}} \cap \ldots \cap A_{j_{k}}$ equals zero.

Theorem A (Comparison Theorem, [2], Th. 5.9). Let $n$-tuples of plurisubharmonic functions $u_{1}, \ldots, u_{n}$ and $v_{1}, \ldots, v_{n}$ be in general position on a neighbourhood of a point $x \in \mathbb{C}^{n}$. Suppose that $u_{j}(x)=-\infty, 1 \leq j \leq n$, and

$$
\limsup _{z \rightarrow x} \frac{v_{j}(z)}{u_{j}(z)}=l_{j}<\infty
$$


Then

$$
\left.d d^{c} v_{1} \wedge \ldots \wedge d d^{c} v_{n}\right|_{\{x\}} \leq\left. l_{1} \ldots l_{n} d d^{c} u_{1} \wedge \ldots \wedge d d^{c} u_{n}\right|_{\{x\}} .
$$

We also obtain a geometric interpretation for the value $N(u, x)$ (Theorem 7). Let $\Theta_{u, x}$ be the set of points $b \in \overline{\mathbb{R}_{+}^{n}}$ such that $\nu(u, x, a) \geq\langle b, a\rangle$ for some $a \in \mathbb{R}_{+}^{n}$. Then

$$
\tau(u, x) \geq N(u, x)=n ! \operatorname{Vol}\left(\Theta_{u, x}\right) .
$$

In many cases the volume of $\Theta_{u, x}$ can be easily calculated, so (6) gives an effective formula for $N(u, x)$.

To illustrate these results, consider functions $u=\log |f|, f=\left(f_{1}, \ldots, f_{n}\right)$ being an equidimensional holomorphic mapping with an isolated zero at a point $x$. It is probably the only class of functions whose residual measures were studied in detail before. In this case, $\tau(u, x)$ equals $m$, the multiplicity of $f$ at $x$, and

$$
\nu(\log |f|, x, a)=I(f, x, a):=\inf \left\{\langle a, p\rangle: p \in \omega_{x}\right\}
$$

where

$$
\omega_{x}=\left\{p \in \mathbb{Z}_{+}^{n}: \sum_{j}\left|\frac{\partial^{p} f_{j}}{\partial z^{p}}(x)\right| \neq 0\right\}
$$

(see [13]). For polynomials $F: \mathbb{C}^{n} \rightarrow \mathbb{C}$, the value $I(F, x, a)$ is a known object (the index of $F$ at $x$ with respect to the weight $a$ ) used in number theory (see e.g. [11]).

Relation (4) gives us $m=\tau(\log |f|, x) \geq N(\log |f|, x)$. In general, the value $N(\log |f|, x)$ is not comparable to $m_{1} \ldots m_{n}$ with $m_{j}$ the multiplicity of the function $f_{j}$ : for $f(z)=\left(z_{1}^{2}+z_{2}, z_{2}\right)$ and $x=0, m_{1} m_{2}=1<2=$ $N(\log |f|, x)=m$ while for $f(z)=\left(z_{1}^{2}+z_{2}, z_{2}^{3}\right), N(\log |f|, x)=2<3=$ $m_{1} m_{2}<6=m$. A sharper bound for $m$ can be obtained from (5) with $u_{j}=\log \left|f_{j}\right|, 1 \leq j \leq n$. In this case, the left-hand side of (5) equals $m$, and its right-hand side with $a_{1}=\ldots=a_{n}$ equals $m_{1} \ldots m_{n}$. For both the above examples of the mapping $f$, the supremum of the right-hand side of (5) over $a \in \mathbb{R}_{+}^{n}$ equals $m$. For $a_{1}, \ldots, a_{n}$ rational, relation (5) is a known bound for $m$ via the multiplicities of weighted homogeneous initial Taylor polynomials of $f_{j}$ with respect to the weights $\left(a_{1}, \ldots, a_{n}\right)$ ([1], Th. 22.7).

Recall that the convex hull $\Gamma_{+}(f, x)$ of the set $\bigcup_{p}\left\{p+\mathbb{R}_{+}^{n}\right\}, p \in \omega_{x}$, is called the Newton polyhedron of $\left(f_{1}, \ldots, f_{n}\right)$ at $x$, the union $\Gamma(f, x)$ of the compact faces of the boundary of $\Gamma_{+}(f, x)$ is called the Newton boundary of $\left(f_{1}, \ldots, f_{n}\right)$ at $x$, and the value $N_{f, x}=n ! \operatorname{Vol}\left(\Gamma_{-}(f, x)\right)$ with $\Gamma_{-}(f, x)=$ $\{\lambda t: t \in \Gamma(f, x), 0 \leq \lambda \leq 1\}$ is called the Newton number of $\left(f_{1}, \ldots, f_{n}\right)$ at $x$ (see [10], [1]). The relation

$$
m \geq N_{f, x}
$$


was established by A. G. Kouchnirenko [9] (see also [1], Th. 22.8). Since $\Theta_{\log |f|, x}=\Gamma_{-}(f, x),(8)$ is a particular case of $(6)$. It is the reason for calling $N(u, x)$ the Newton number of $u$ at $x$.

These observations show that the technique of plurisubharmonic functions (and local indicators in particular) is quite a powerful tool to produce, in a unified and simple way, sharp bounds for the multiplicities of holomorphic mappings.

Finally, we obtain a description for the indicator $\Psi_{u, x}(z)$ as the weak limit of the functions $m^{-1} u\left(x_{1}+z_{1}^{m}, \ldots, x_{n}+z_{n}^{m}\right)$ as $m \rightarrow \infty$ (Theorem 8), so $\Psi_{u, x}$ can be viewed as the tangent (in the logarithmic coordinates) for the function $u$ at $x$. Using this approach we obtain a sufficient condition, in terms of $\mathcal{C}_{n-1}$-capacity, for the residual mass $\tau(u, x)$ to coincide with the Newton number of $u$ at $x$ (Theorem 9).

2. Indicators and their masses. We will use the following notations. For a domain $\Omega$ of $\mathbb{C}^{n}, \operatorname{PSH}(\Omega)$ will denote the class of all plurisubharmonic functions on $\Omega$, PSH $-(\Omega)$ the subclass of nonpositive functions, and $\operatorname{PSH}(\Omega, x)=\operatorname{PSH}(\Omega) \cap L_{\mathrm{loc}}^{\infty}(\Omega \backslash\{x\})$ with $x \in \Omega$.

Let $D=\left\{z \in \mathbb{C}^{n}:\left|z_{k}\right|<1,1 \leq k \leq n\right\}$ be the unit polydisk, $D^{*}=\left\{z \in D: z_{1} \cdot \ldots \cdot z_{n} \neq 0\right\}$, and $\mathbb{R}_{ \pm}^{n}=\left\{t \in \mathbb{R}^{n}: \pm t_{k}>0\right\}$. By CNVI_ $\left(\mathbb{R}_{-}^{n}\right)$ we denote the collection of all nonpositive convex functions on $\mathbb{R}_{-}^{n}$ increasing in each variable $t_{k}$. The mapping $\log : D^{*} \rightarrow \mathbb{R}_{-}^{n}$ is defined as $\log (z)=\left(\log \left|z_{1}\right|, \ldots, \log \left|z_{n}\right|\right)$, and $\operatorname{Exp}: \mathbb{R}_{-}^{n} \rightarrow D^{*}$ is given by $\operatorname{Exp}(t)=\left(\exp t_{1}, \ldots, \exp t_{n}\right)$.

A function $u$ on $D^{*}$ is called $n$-circled if

$$
u(z)=u\left(\left|z_{1}\right|, \ldots,\left|z_{n}\right|\right),
$$

i.e. if $\log ^{*} \operatorname{Exp}^{*} u=u$. Any $n$-circled function $u \in \mathrm{PSH}_{-}\left(D^{*}\right)$ has a unique extension to the whole polydisk $D$ keeping the property (9). The class of such functions will be denoted by $\mathrm{PSH}_{-}^{c}(D)$. The cones CNVI_ $\left(\mathbb{R}_{-}^{n}\right)$ and $\mathrm{PSH}_{-}^{c}(D)$ are isomorphic: $u \in \mathrm{PSH}_{-}^{c}(D) \Leftrightarrow \operatorname{Exp}^{*} u \in \mathrm{CNVI}_{-}\left(\mathbb{R}_{-}^{n}\right), h \in$ $\mathrm{CNVI}_{-}\left(\mathbb{R}_{-}^{n}\right) \Leftrightarrow \log ^{*} h \in \mathrm{PSH}_{-}^{c}(D)$.

Definition 2 (see [15]). A function $\Psi \in \mathrm{PSH}_{-}^{c}(D)$ is called an indicator if its convex image $\operatorname{Exp}^{*} \Psi$ satisfies

$$
\operatorname{Exp}^{*} \Psi(c t)=c \operatorname{Exp}^{*} \Psi(t) \quad \forall c>0, \forall t \in \mathbb{R}_{-}^{n} .
$$

The collection of all indicators will be denoted by $I$. It is a convex subcone of $\mathrm{PSH}_{-}^{c}(D)$, closed in $\mathcal{D}^{\prime}$ (or equivalently, in $L_{\text {loc }}^{1}(D)$ ). Moreover, if $\Psi_{1}$, $\Psi_{2} \in I$ then also $\sup \left\{\Psi_{1}, \Psi_{2}\right\} \in I$.

Every indicator is locally bounded in $D^{*}$. In what follows we will often consider indicators locally bounded in $D \backslash\{0\}$; the class of such indicators will be denoted by $I_{0}: I_{0}=I \cap \operatorname{PSH}(D, 0)$. 
An example of indicators can be given by the functions

$$
\varphi_{a}(z)=\sup _{k} a_{k} \log \left|z_{k}\right|, \quad a_{k} \geq 0,
$$

("simple" indicators). If all $a_{k}>0$, then $\varphi_{a} \in I_{0}$.

Proposition 1. Let $\Psi \in I_{0}, \Psi \neq \equiv 0$. Then

(a) there exist reals $\nu_{1}, \ldots, \nu_{n}>0$ such that

$$
\Psi(z) \geq \varphi_{\nu}(z) \quad \forall z \in D
$$

with $\varphi_{\nu}$ the simple indicator corresponding to $\nu=\left(\nu_{1}, \ldots, \nu_{n}\right)$;

(b) $\Psi \in C(\bar{D} \backslash\{0\}),\left.\Psi\right|_{\partial D}=0$;

(c) the directional Lelong numbers $\nu(\Psi, 0, a)$ of $\Psi$ at the origin with respect to $a \in \mathbb{R}_{+}^{n}$ (see (3)) are

$$
\nu(\Psi, 0, a)=-\Psi(\operatorname{Exp}(-a)),
$$

and its Lelong number is $\nu(\Psi, 0)=-\Psi\left(e^{-1}, \ldots, e^{-1}\right)$;

(d) $\left(d d^{c} \Psi\right)^{n}=0$ on $D \backslash\{0\}$.

P r o o f. Let $\Psi_{k}\left(z_{k}\right)$ denote the restriction of the indicator $\Psi(z)$ to the disk $D^{(k)}=\left\{z \in D: z_{j}=0 \forall j \neq k\right\}$. By monotonicity of $\operatorname{Exp}^{*} \Psi, \Psi(z) \geq \Psi_{k}\left(z_{k}\right)$. Since $\Psi_{k}$ is a nonzero indicator in the disk $D^{(k)} \subset \mathbb{C}, \Psi_{k}\left(z_{k}\right)=\nu_{k} \log \left|z_{k}\right|$ with some $\nu_{k}>0$, and (a) follows.

As $\operatorname{Exp}^{*} \Psi \in C\left(\mathbb{R}_{-}^{n}\right)$, we have $\Psi \in C\left(D^{*}\right)$. Its continuity in $D \backslash\{0\}$ can be shown by induction on $n$. For $n=1$ it is obvious, so assuming it for $n \leq l$, consider any point $z^{0} \neq 0$ with $z_{j}^{0}=0$. Let $z^{s} \rightarrow z^{0}$; then the points $\widetilde{z}^{s}$ with $\widetilde{z}_{j}^{s}=0$ and $\widetilde{z}_{m}^{s}=z_{m}^{s}, m \neq j$, also tend to $z^{0}$, and by the induction hypothesis, $\Psi\left(\widetilde{z}^{s}\right) \rightarrow \Psi\left(\widetilde{z}^{0}\right)=\Psi\left(z^{0}\right)$. So, $\liminf _{s \rightarrow \infty} \Psi\left(z^{s}\right) \geq$ $\lim _{s \rightarrow \infty} \Psi\left(\widetilde{z}^{s}\right)=\Psi\left(z^{0}\right)$, i.e. $\Psi$ is lower semicontinuous and hence continuous at $z^{0}$. Continuity of $\Psi$ up to $\partial D$ and the boundary condition follow from (11).

Equality (12) is an immediate consequence of the definition of the directional Lelong numbers (3) and the homogeneity condition (10). The relation $\nu(u, x)=\nu(u, x,(1, \ldots, 1))[5]$ gives us the desired expression for $\nu(\Psi, 0)$.

Finally, statement (d) follows from the homogeneity condition (10) (see [15], Proposition 4).

For functions $\Psi \in I_{0}$, the complex Monge-Ampère operator $\left(d d^{c} \Psi\right)^{n}$ is well defined and gives a nonnegative measure on $D$. By Proposition 1,

$$
\left(d d^{c} \Psi\right)^{n}=\tau(\Psi) \delta(0)
$$

with some constant $\tau(\Psi) \geq 0$ which is strictly positive unless $\Psi \equiv 0$. In this section, we will study the value $\tau(\Psi)$.

An upper bound for $\tau(\Psi)$ is given by 
Proposition 2. For every $\Psi \in I_{0}$,

$$
\tau(\Psi) \leq \nu_{1} \ldots \nu_{n}
$$

with $\nu_{1}, \ldots, \nu_{n}$ as in Proposition 1(a).

Proof. Since all $\nu_{k}>0$, the simple indicator $\varphi_{\nu}$ is in $I_{0}$, and (11) implies

$$
\limsup _{z \rightarrow 0} \frac{\Psi(z)}{\varphi_{\nu}(z)} \leq 1
$$

so (13) follows by Theorem A.

To obtain a lower bound for $\tau(\Psi)$, we need a relation between $\Psi(z)$ and $\Psi\left(z^{0}\right)$ for $z, z^{0} \in D$. Define

$$
\Phi\left(z, z^{0}\right)=\sup _{k} \frac{\log \left|z_{k}\right|}{|\log | z_{k}^{0}||}, \quad z \in D, z^{0} \in D^{*} .
$$

When considered as a function of $z$ with $z^{0}$ fixed, $\Phi\left(z, z^{0}\right)$ is in $I_{0}$.

Proposition 3. For any $\Psi \in I$, we have $\Psi(z) \leq\left|\Psi\left(z^{0}\right)\right| \Phi\left(z, z^{0}\right)$ for all $z \in D, z^{0} \in D^{*}$.

Proof. For a fixed $z^{0} \in D^{*}$ and $t^{0}=\log \left(z^{0}\right)$, define $u=\left|\Psi\left(z^{0}\right)\right|^{-1} \operatorname{Exp}^{*} \Psi$ and $v=\operatorname{Exp}^{*} \Phi=\sup _{k} t_{k} /\left|t_{k}^{0}\right|$. It suffices to establish the inequality $u(t) \leq$ $v(t)$ for all $t \in \mathbb{R}_{-}^{n}$ with $t_{k}^{0}<t_{k}<0,1 \leq k \leq n$. Given such a $t$, define $\lambda_{0}=[1+v(t)]^{-1}$. Since $\left\{t^{0}+\lambda\left(t-t^{0}\right): 0 \leq \lambda \leq \lambda_{0}\right\} \subset \overline{\mathbb{R}_{-}^{n}}$, the functions $u_{t}(\lambda):=u\left(t^{0}+\lambda\left(t-t^{0}\right)\right)$ and $v_{t}(\lambda):=v\left(t^{0}+\lambda\left(t-t^{0}\right)\right)$ are well defined on $\left[0, \lambda_{0}\right]$. Furthermore, $u_{t}$ is convex and $v_{t}$ is linear there, $u_{t}(0)=v_{t}(0)=-1$, $u_{t}\left(\lambda_{0}\right) \leq v_{t}\left(\lambda_{0}\right)=0$. This implies $u_{t}(\lambda) \leq v_{t}(\lambda)$ for all $\lambda \in\left[0, \lambda_{0}\right]$. In particular, as $\lambda_{0}>1, u(t)=u_{t}(1) \leq v_{t}(1)=v(t)$, which completes the proof.

Consider now the function

$$
P(z)=-\prod_{1 \leq k \leq n}|\log | z_{k} \|^{1 / n} \in I .
$$

TheOREM 1. The Monge-Ampère mass $\tau(\Psi)$ of any indicator $\Psi \in I_{0}$ has the bound

$$
\tau(\Psi) \geq\left|\frac{\Psi\left(z^{0}\right)}{P\left(z^{0}\right)}\right|^{n} \quad \forall z^{0} \in D^{*}
$$

where the function $P$ is defined by (14).

Pro of. By Proposition 3,

$$
\frac{\Psi(z)}{\Phi\left(z, z^{0}\right)} \leq\left|\Psi\left(z^{0}\right)\right| \quad \forall z \in D, z^{0} \in D^{*} .
$$

By Theorem A,

$$
\left(d d^{c} \Psi\right)^{n} \leq\left|\Psi\left(z^{0}\right)\right|^{n}\left(d d^{c} \Phi\left(z, z^{0}\right)\right)^{n},
$$


and the statement follows from the fact that

$$
\left(d d^{c} \Phi\left(z, z^{0}\right)\right)^{n}=\left.\prod_{1 \leq k \leq n}|\log | z_{k}^{0}\right|^{-1}=\left|P\left(z^{0}\right)\right|^{-n} .
$$

Remarks. 1. One can consider the value

$$
A_{\Psi}=\sup _{z \in D}\left|\frac{\Psi(z)}{P(z)}\right|^{n}
$$

by Theorem 1 ,

$$
\tau(\Psi) \geq A_{\Psi}
$$

2. Let $I_{0, M}=\left\{\Psi \in I_{0}: \tau(\Psi) \leq M\right\}, M>0$. Then (15) gives a lower bound for the class $I_{0, M}$ :

$$
\Psi(z) \geq M^{1 / n} P(z) \quad \forall z \in D, \forall \Psi \in I_{0, M} .
$$

Let now $\Psi_{1}, \ldots, \Psi_{n} \in I$ be in general position in the sense of Definition 1 . Then the current $\bigwedge_{k} d d^{c} \Psi_{k}$ is well defined, as is $\left(d d^{c} \Psi\right)^{n}$ with $\Psi=\sup _{k} \Psi_{k}$. Moreover, we have

Proposition 4. If $\Psi_{1}, \ldots, \Psi_{n} \in I$ are in general position, then

$$
\bigwedge_{k} d d^{c} \Psi_{k}=0 \quad \text { on } D \backslash\{0\} \text {. }
$$

Pr o o f. For $\Psi_{1}, \ldots, \Psi_{n} \in I_{0}$, the statement follows from Proposition $1(\mathrm{~d})$ and the polarization formula

$$
\bigwedge_{k} d d^{c} \Psi_{k}=\frac{(-1)^{n}}{n !} \sum_{j=1}^{n}(-1)^{j} \sum_{1 \leq i_{1}<\ldots<i_{j} \leq n}\left(d d^{c} \sum_{k=1}^{j} \Psi_{j_{k}}\right)^{n} .
$$

When the only condition on $\left\{\Psi_{k}\right\}$ is to be in general position, we can replace $\Psi_{k}(z)$ with $\Psi_{k, N}(z)=\sup \left\{\Psi_{k}(z), N \sup _{j} \log \left|z_{j}\right|\right\} \in I_{0}$ for which $\bigwedge_{k} d d^{c} \Psi_{k, N}=0$ on $D \backslash\{0\}$. Since $\Psi_{k, N} \backslash \Psi_{k}$ as $N \rightarrow \infty$, this gives us (18).

The mass of $\bigwedge_{k} d d^{c} \Psi_{k}$ will be denoted by $\tau\left(\Psi_{1}, \ldots, \Psi_{n}\right)$.

Theorem 2. Let $\Psi_{1}, \ldots, \Psi_{n} \in I$ be in general position, $\Psi=\sup _{k} \Psi_{k}$. Then

(a) $\tau(\Psi) \leq \tau\left(\Psi_{1}, \ldots, \Psi_{n}\right)$;

(b) $\tau\left(\Psi_{1}, \ldots, \Psi_{n}\right) \geq\left|P\left(z^{0}\right)\right|^{-n} \prod_{k}\left|\Psi_{k}\left(z^{0}\right)\right|$ for all $z^{0} \in D^{*}$, the function $P$ being defined by (14).

P r o of. Since

$$
\frac{\Psi(z)}{\Psi_{k}(z)} \leq 1 \quad \forall z \neq 0
$$

statement (a) follows from Theorem A. 
Statement (b) results from Proposition 3 exactly as the statement of Theorem 1 does.

3. Geometric interpretation. In this section we study the masses $\tau(\Psi)$ of indicators $\Psi \in I_{0}$ by means of their convex images $\operatorname{Exp}^{*} \Psi \in \mathrm{CNVI}_{-}\left(\mathbb{R}_{-}^{n}\right)$.

Let $V \in \mathrm{PSH}_{-}^{c}(r D) \cap C^{2}(r D), r<1$, and $v=\operatorname{Exp}^{*} V \in \mathrm{CNVI}_{-}\left(\left(\mathbb{R}_{-}+\right.\right.$ $\left.\log r)^{n}\right)$. Since

$$
\frac{\partial^{2} V(z)}{\partial z_{j} \partial \bar{z}_{k}}=\left.\frac{1}{4 z_{j} \bar{z}_{k}} \cdot \frac{\partial^{2} v(t)}{\partial t_{j} \partial t_{k}}\right|_{t=\log (z)}, \quad z \in r D^{*},
$$

we have

$$
\operatorname{det}\left(\frac{\partial^{2} V(z)}{\partial z_{j} \partial \bar{z}_{k}}\right)=\left.4^{-n}\left|z_{1} \ldots z_{n}\right|^{-2} \operatorname{det}\left(\frac{\partial^{2} v(t)}{\partial t_{j} \partial t_{k}}\right)\right|_{t=\log (z)} .
$$

By setting $z_{j}=\exp \left\{t_{j}+i \theta_{j}\right\}, 0 \leq \theta \leq 2 \pi$, we get $\beta_{n}(z)=\left|z_{1} \ldots z_{n}\right|^{2} d t d \theta$, so

$$
\left(d d^{c} V\right)^{n}=n !\left(\frac{2}{\pi}\right)^{n} \operatorname{det}\left(\frac{\partial^{2} V}{\partial z_{j} \partial \bar{z}_{k}}\right) \beta_{n}=\frac{n !}{(2 \pi)^{n}} \operatorname{det}\left(\frac{\partial^{2} v}{\partial t_{j} \partial t_{k}}\right) d t d \theta
$$

Every function $U \in \mathrm{PSH}_{-}^{c}(D) \cap L^{\infty}(D)$ is the limit of a decreasing sequence of functions $U_{l} \in \mathrm{PSH}_{-}^{c}(E) \cap C^{2}(E)$ on an $n$-circled domain $E \subset \subset D$, and by the convergence theorem for the complex Monge-Ampère operators,

$$
\left.\left.\left(d d^{c} U_{l}\right)^{n}\right|_{E} \rightarrow\left(d d^{c} U\right)^{n}\right|_{E} .
$$

On the other hand, for $u_{l}=\operatorname{Exp}^{*} U_{l}$ and $u=\operatorname{Exp}^{*} U$,

$$
\left.\left.\operatorname{det}\left(\frac{\partial^{2} u_{l}}{\partial t_{j} \partial t_{k}}\right) d t\right|_{\log \left(D^{*} \cap E\right)} \rightarrow \mathcal{M A}[u]\right|_{\log \left(D^{*} \cap E\right)},
$$

the real Monge-Ampère operator of $u$ (see [16]).

Since $\left(d d^{c} U_{l}\right)^{n}$ and $\left(d d^{c} U\right)^{n}$ cannot charge pluripolar sets, (20) with $V=U_{l}$ and (21), (22) imply

$$
\left(d d^{c} U\right)^{n}(E)=n !(2 \pi)^{-n} \mathcal{M A}[u] d \theta\left(\log (E) \times[0,2 \pi]^{n}\right)
$$

for any $n$-circled Borel set $E \subset D$, i.e.

$$
\left(d d^{c} U\right)^{n}(E)=n ! \mathcal{M} \mathcal{A}[u](\log (E)) .
$$

This relation allows us to calculate $\tau(\Psi)$ by using the technique of real Monge-Ampère operators in $\mathbb{R}^{n}$ (see [16]).

Let $\Psi \in I$. Consider the set

$$
B_{\Psi}=\left\{a \in \mathbb{R}_{+}^{n}:\langle a, t\rangle \leq \operatorname{Exp}^{*} \Psi(t) \forall t \in \mathbb{R}_{-}^{n}\right\}
$$

and define

$$
\Theta_{\Psi}=\overline{\mathbb{R}_{+}^{n} \backslash B_{\Psi}}
$$


Clearly, the set $B_{\Psi}$ is convex, so $\operatorname{Exp}^{*} \Psi$ is the restriction of its support function to $\mathbb{R}_{-}^{n}$. If $\Psi \in I_{0}$, the set $\Theta_{\Psi}$ is bounded. Indeed, $a \in \Theta_{\Psi}$ if and only if $\left\langle a, t^{0}\right\rangle \geq \operatorname{Exp}^{*} \Psi\left(t^{0}\right)$ for some $t^{0} \in \mathbb{R}_{-}^{n}$, which implies $\left|a_{j}\right| \leq\left|\operatorname{Exp}^{*} \Psi\left(t^{0}\right) / t_{j}^{0}\right|$ for all $j$. By Proposition 1(a), $\left|\operatorname{Exp}^{*} \Psi\left(t^{0}\right)\right| \leq \nu_{j}\left|t_{j}\right|$ and therefore $\left|a_{j}\right| \leq \nu_{j}$ for all $j$.

Given a set $F \subset \mathbb{R}^{n}$, we denote its Euclidean volume by $\operatorname{Vol}(F)$.

TheOREM 3. For any indicator $\Psi \in I_{0}$, we have the relation

$$
\tau(\Psi)=n ! \operatorname{Vol}\left(\Theta_{\Psi}\right)
$$

with the set $\Theta_{\Psi}$ given by (24) and (25).

Proof. Define $U(z)=\sup \{\Psi(z),-1\} \in \mathrm{PSH}_{-}^{c}(D) \cap C(D), u=\operatorname{Exp}^{*} U$ $\in \mathrm{CNVI}_{-}\left(\mathbb{R}_{-}^{n}\right)$. Since $U(z)=\Psi(z)$ near $\partial D$,

$$
\tau(\Psi)=\int_{D}\left(d d^{c} U\right)^{n}
$$

Furthermore, as $\left(d d^{c} U\right)^{n}=0$ outside the set $E=\{z \in D: \Psi(z)=-1\}$,

$$
\tau(\Psi)=\int_{E}\left(d d^{c} U\right)^{n}
$$

In view of $(23)$,

$$
\int_{E}\left(d d^{c} U\right)^{n}=n ! \int_{\log (E)} \mathcal{M A}[u] .
$$

As was shown in [16], for any convex function $v$ in a domain $\Omega \subset \mathbb{R}^{n}$,

$$
\int_{F} \mathcal{M A}[v]=\operatorname{Vol}(\omega(F, v)) \quad \forall F \subset \Omega,
$$

where

$$
\omega(F, v)=\bigcup_{t^{0} \in F}\left\{a \in \mathbb{R}^{n}: v(t) \geq v\left(t^{0}\right)+\left\langle a, t-t^{0}\right\rangle \forall t \in \Omega\right\}
$$

is the gradient image of the set $F$ for the surface $\{y=v(x): x \in \Omega\}$.

We claim that

$$
\Theta_{\Psi}=\omega(\log (E), u)
$$

Observe that

$$
\Theta_{\Psi}=\left\{a \in \overline{\mathbb{R}_{+}^{n}}: \sup _{\psi(t)=-1}\langle a, t\rangle \geq-1\right\} \quad \text { where } \quad \psi=\operatorname{Exp}^{*} \Psi .
$$

If $a \in \omega(\log (E), u)$, then for some $t^{0} \in \mathbb{R}_{-}^{n}$ with $\psi\left(t^{0}\right)=1$ we have $\left\langle a, t^{0}\right\rangle \geq\langle a, t\rangle$ for all $t \in \mathbb{R}_{-}^{n}$ such that $\psi(t)<-1$. Taking here $t_{j} \rightarrow-\infty$ we get $a_{j} \geq 0$, i.e. $a \in \overline{\mathbb{R}_{+}^{n}}$. Moreover, $\left\langle a, t^{0}\right\rangle \geq\langle a, t\rangle-1-\psi(t)$ for all $t \in \mathbb{R}_{-}^{n}$ with $\psi(t)>-1$, and letting $t \rightarrow 0$ we derive $\left\langle a, t^{0}\right\rangle \geq-1$. Therefore, $a \in \Theta_{\Psi}$ and $\Theta_{\Psi} \supset \omega(\log (E), u)$. 
Now we prove the converse inclusion. If $a \in \Theta_{\Psi} \cap \mathbb{R}_{+}^{n}$, then

$$
\sup \left\{\left\langle a, t^{0}\right\rangle: t^{0} \in \log (E)\right\} \geq-1 .
$$

Let $t$ be such that $\psi(t)=-\delta>-1$. Then $t / \delta \in \log (E)$ and thus

$$
\begin{aligned}
\langle a, t\rangle-1-\psi(t) & =\delta\langle a, t / \delta\rangle-1+\delta \leq \delta \sup _{t^{0} \in \log (E)}\left\langle a, t^{0}\right\rangle-1+\delta \\
& \leq \sup _{t^{0} \in \log (E)}\left\langle a, t^{0}\right\rangle=\sup _{z^{0} \in E}\left\langle a, \log \left(z^{0}\right)\right\rangle .
\end{aligned}
$$

Since $E$ is compact, the latter supremum is attained at some point $\widehat{z}^{0}$. Furthermore, $\widehat{z}^{0} \in E \cap D^{*}$ because $a_{k} \neq 0,1 \leq k \leq n$. Hence $\sup _{t^{0} \in \log (E)}\left\langle a, t^{0}\right\rangle$ $=\left\langle a, \widehat{t}^{0}\right\rangle$ with $\widehat{t}^{0}=\log \left(z^{0}\right) \in \mathbb{R}_{-}^{n}$, so that $a \in \omega(\log (E), u)$ and $\Theta_{\Psi} \cap \mathbb{R}_{+}^{n} \subset$ $\omega(\log (E), u)$. Since $\omega(\log (E), u)$ is closed, this implies $\Theta_{\Psi}=\omega(\log (E), u)$, and (30) follows.

Now relation (26) is a consequence of (27)-(30). The theorem is proved.

Note that the value $\tau\left(\Psi_{1}, \ldots, \Psi_{n}\right)$ can also be expressed in geometric terms. Namely, if $\Psi_{1}, \ldots, \Psi_{n} \in I_{0}$, the polarization formula (19) gives us, by Theorem 3,

$$
\tau\left(\Psi_{1}, \ldots, \Psi_{n}\right)=(-1)^{n} \sum_{j=1}^{n}(-1)^{j} \sum_{1 \leq i_{1}<\ldots<i_{j} \leq n} \operatorname{Vol}\left(\Theta_{\sum_{k} \Psi_{j_{k}}}\right) .
$$

We can also give an interpretation for the bound (17). Write $A_{\Psi}$ from (16) as

$$
A_{\Psi}=\sup _{a \in \mathbb{R}_{+}^{n}} \frac{|\psi(-a)|^{n}}{a_{1} \ldots a_{n}}=\sup _{a \in \mathbb{R}_{+}^{n}}\left|\psi\left(-a / a_{1}\right) \ldots \psi\left(-a / a_{n}\right)\right|,
$$

where $\psi=\operatorname{Exp}^{*} \Psi$. For any $a \in \mathbb{R}_{+}^{n}$, the point $a^{(j)}$ whose $j$ th coordinate equals $\left|\psi\left(-a / a_{j}\right)\right|$ and the others are zero, has the property $\left\langle a^{(j)},-a\right\rangle=$ $\psi(-a)$. This remains true for every convex combination $\sum \varrho_{j} a^{(j)}$, and thus $r \sum \varrho_{j} a^{(j)} \in \Theta_{\Psi}$ with any $r \in[0,1]$. Since $(n !)^{-1}\left|\psi\left(-a / a_{1}\right) \ldots \psi\left(-a / a_{n}\right)\right|$ is the volume of the simplex generated by the points $0, a^{(1)}, \ldots, a^{(n)}$, we see from (31) that $(n !)^{-1} A_{\Psi}$ is the supremum of the volumes of all simplices contained in $\Theta_{\Psi}$.

Moreover, $(n !)^{-1}[\nu(\Psi, 0)]^{n}$ is the volume of the simplex

$$
\left\{a \in \overline{\mathbb{R}_{+}^{n}}:\langle a,(1, \ldots, 1)\rangle \leq \nu(\Psi, 0)\right\} \subset \Theta_{\Psi} .
$$

This is a geometric description for the "standard" bound $\tau(\Psi) \geq[\nu(\Psi, 0)]^{n}$.

4. Singularities of plurisubharmonic functions. Let $u$ be a plurisubharmonic function in a domain $\Omega \subset \mathbb{C}^{n}$, and $\nu(u, x, a)$ be its directional Lelong number (3) at $x \in \Omega$ with respect to $a \in \mathbb{R}_{+}^{n}$. Fix a point $x$. It is 
known [5] that the function $a \mapsto \nu(u, x, a)$ is concave on $\mathbb{R}_{+}^{n}$. So, the function

$$
\psi_{u, x}(t):=-\nu(u, x,-t), \quad t \in \mathbb{R}_{-}^{n},
$$

belongs to $\mathrm{CNVI}_{-}\left(\mathbb{R}_{-}^{n}\right)$ and thus

$$
\Psi_{u, x}:=\log ^{*} \psi_{u, x} \in \mathrm{PSH}_{-}^{c}(D) .
$$

Moreover, due to the positive homogeneity of $\nu(u, x, a)$ in $a, \Psi_{u, x} \in I$. The function $\Psi_{u, x}$ was introduced in [15] and called the (local) indicator of $u$ at $x$. According to (3),

$$
\begin{aligned}
\Psi_{u, x}(z) & =\lim _{R \rightarrow \infty} R^{-1} \sup \left\{u(y):\left|y_{k}-x_{k}\right| \leq\left|z_{k}\right|^{R}, 1 \leq k \leq n\right\} \\
& =\lim _{R \rightarrow \infty} R^{-1} \frac{1}{(2 \pi)^{n}} \int_{[0,2 \pi]^{n}} u\left(x_{k}+\left|z_{k}\right|^{R} e^{i \theta_{k}}\right) d \theta_{1} \ldots d \theta_{n} .
\end{aligned}
$$

Clearly, $\Psi_{u, x} \equiv 0$ if and only if $\nu(u, x)=0$. It is easy to see that $\Psi_{\Phi, 0}=\Phi$ for any $\Phi \in I$. In particular,

$$
\nu(u, x, a)=\nu\left(\Psi_{u, x}, 0, a\right)=-\Psi_{u, x}(\operatorname{Exp}(-a)) \quad \forall a \in \mathbb{R}_{+}^{n} .
$$

So, the results of the previous sections can be applied to study directional Lelong numbers of arbitrary plurisubharmonic functions.

Proposition 5 (cf. [7], Prop. 5.3). For any $u \in \operatorname{PSH}(\Omega)$,

$$
\nu(u, x, a) \geq \nu(u, x, b) \min _{k} \frac{a_{k}}{b_{k}} \quad \forall x \in \Omega, \forall a, b \in \mathbb{R}_{+}^{n} .
$$

P r o of. In view of (32), this follows from Proposition 3.

For $r \in \mathbb{R}_{+}^{n}$ and $z \in \mathbb{C}^{n}$, we set $r^{-1}=\left(r_{1}^{-1}, \ldots, r_{n}^{-1}\right)$ and $r \cdot z=$ $\left(r_{1} z_{1}, \ldots, r_{n} z_{n}\right)$.

Proposition 6 ([15]). Any function $u \in \operatorname{PSH}(\Omega)$ has the bound

$$
u(z) \leq \Psi_{u, x}\left(r^{-1} \cdot z\right)+\sup \left\{u(y): y \in D_{r}(x)\right\}
$$

for all $z \in D_{r}(x)=\left\{y:\left|y_{k}-x_{k}\right| \leq r_{k}, 1 \leq k \leq n\right\} \subset \subset \Omega$.

Proof. Assume for simplicity $x=0, D_{r}(0)=D_{r}$.

Consider the function $v(z)=u(r \cdot z)-\sup \left\{u(y): y \in D_{r}\right\} \in$ PSH_$_{-}(D)$. The function $g_{v}(R, t):=\sup \left\{v(z):\left|z_{k}\right| \leq \exp \left\{R t_{k}\right\}, 1 \leq k \leq n\right\}$ is convex in $R>0$ and $t \in \mathbb{R}_{-}^{n}$, so as $R \rightarrow \infty$,

$$
\frac{g_{v}(R, t)-g_{v}\left(R_{1}, t\right)}{R-R_{1}} \nearrow \psi_{v, 0}(t),
$$

where $\psi_{v, 0}=\operatorname{Exp}^{*} \Psi_{v, 0}$.

For $R=1$ and $R_{1} \rightarrow 0,(34)$ gives us $g_{v}(1, t) \leq \psi_{v, 0}(t)$ and thus (33). The proposition is proved. 
Let $\Omega_{k}(x)$ be the connected component of the set $\Omega \cap\left\{z \in \mathbb{C}^{n}: z_{j}=x_{j}\right.$ $\forall j \neq k\}$ containing the point $x$. If for some $x \in \Omega,\left.u\right|_{\Omega_{k}(x)} \not \equiv-\infty$ for all $k$, then $\Psi_{u, x} \in I_{0}$. For example, this is fulfilled for $u \in \operatorname{PSH}(\Omega, x)$.

If $u \in \operatorname{PSH}(\Omega, x)$, the measure $\left(d d^{c} u\right)^{n}$ is defined on $\Omega$. Its residual mass at $x$ will be denoted by $\tau(u, x)$ :

$$
\tau(u, x)=\left.\left(d d^{c} u\right)^{n}\right|_{\{x\}} \cdot
$$

The indicator $\Psi_{u, x}$ of such a function belongs to the class $I_{0}$. Define

$$
N(u, x)=\tau\left(\Psi_{u, x}\right) .
$$

Proposition 7 ([15], Th. 1). If $u \in \operatorname{PSH}(\Omega, x)$, then $\tau(u, x) \geq N(u, x)$.

Proof. Inequality (33) implies

$$
\limsup _{z \rightarrow x} \frac{\Psi_{u, x}\left(r^{-1} \cdot(z-x)\right)}{u(z)} \leq 1
$$

and since

$$
\lim _{y \rightarrow 0} \frac{\left.\Psi_{u, x}\left(r^{-1} \cdot y\right)\right)}{\Psi_{u, x}(y)}=1 \quad \forall r \in \mathbb{R}_{+}^{n},
$$

the statement follows from Theorem A.

So, to estimate $\tau(u, x)$ we may apply the bounds for $\tau\left(\Psi_{u, x}\right)$ from the previous section.

Theorem 4. If $u \in \operatorname{PSH}(\Omega, x)$, then

$$
\tau(u, x) \geq \frac{[\nu(u, x, a)]^{n}}{a_{1} \ldots a_{n}} \quad \forall a \in \mathbb{R}_{+}^{n} ;
$$

in other words, $\tau(u, x) \geq A_{u, x}$ where $A_{u, x}=A_{\Psi_{u, x}}$ is defined by (16).

Pr o of. The result follows from Theorem 1 and Proposition 7.

Let now $u_{1}, \ldots, u_{n} \in \operatorname{PSH}(\Omega)$ be in general position in the sense of Definition 1. Then the current $\bigwedge_{k} d d^{c} u_{k}$ is defined on $\Omega$ ([2], Th. 2.5); denote its residual mass at a point $x$ by $\tau\left(u_{1}, \ldots, u_{n} ; x\right)$. Moreover, the $n$-tuple of their indicators $\Psi_{u_{k}, x}$ is also in general position, which implies

$$
\bigwedge_{k} d d^{c} \Psi_{u_{k}, x}=\tau\left(\Psi_{u_{1}, x}, \ldots, \Psi_{u_{n}, x}\right) \delta(0)
$$

(Proposition 4).

In view of Theorem A and Proposition 6 we have

TheOREM 5. The residual mass $\tau\left(u_{1}, \ldots, u_{n} ; x\right)$ of the current $\bigwedge_{k} d d^{c} u_{k}$ has the bound $\tau\left(u_{1}, \ldots, u_{n} ; x\right) \geq \tau\left(\Psi_{u_{1}, x}, \ldots, \Psi_{u_{n}, x}\right)$.

Now Theorems 2 and 5 give us 
Theorem 6.

$$
\tau\left(u_{1}, \ldots, u_{n} ; x\right) \geq \frac{\prod_{j} \nu\left(u_{j}, x, a\right)}{a_{1} \ldots a_{n}} \quad \forall a \in \mathbb{R}_{+}^{n} .
$$

REMARK. For $a_{1}=\ldots=a_{n}$, inequality (35) is proved in [2], Cor. 5.10.

Finally, by combination of Proposition 7 and Theorem 3 we get

Theorem 7. For any function $u \in \operatorname{PSH}(\Omega, x)$,

$$
\tau(u, x) \geq N(u, x)=n ! V\left(\Theta_{u, x}\right)
$$

with

$$
\Theta_{u, x}=\left\{b \in \mathbb{R}_{+}^{n}: \sup _{\sum a_{k}=1}[\nu(u, x, a)-\langle b, a\rangle] \geq 0\right\} .
$$

Remark on holomorphic mappings. Let $f=\left(f_{1}, \ldots, f_{n}\right)$ be a holomorphic mapping of a neighbourhood $\Omega$ of the origin into $\mathbb{C}^{n}$ and $f(0)=0$ be its isolated zero. Then in a subdomain $\Omega^{\prime} \subset \Omega$ the zero sets $A_{j}$ of the functions $f_{j}$ satisfy the conditions

$$
A_{1} \cap \ldots \cap A_{n} \cap \Omega^{\prime}=\{0\}, \quad \operatorname{codim} A_{j_{1}} \cap \ldots \cap A_{j_{k}} \cap \Omega^{\prime} \geq k
$$

for all choices of indices $j_{1}<\ldots<j_{k}, k \leq n$. Set $u=\log |f|, u_{j}=\log \left|f_{j}\right|$. It is known that $\tau(u, 0)=\tau\left(u_{1}, \ldots, u_{n} ; 0\right)=m_{f}$, the multiplicity of $f$ at 0 . For $a=(1, \ldots, 1), \nu\left(u_{j}, 0, a\right)$ equals $m_{j}$, the multiplicity of $f_{j}$ at 0 . Therefore, (35) with $a=(1, \ldots, 1)$ gives us the standard bound $m_{f} \geq m_{1} \ldots m_{n}$.

For $a_{j}$ rational, (35) is the known estimate of $m_{f}$ via the multiplicities of weighted homogeneous initial Taylor polynomials for $f_{j}$ (see e.g. [1], Th. 22.7). Indeed, due to the positive homogeneity of the directional Lelong numbers, we can take $a_{j} \in \mathbb{Z}_{+}^{n}$. Then by $(7), \nu\left(u_{j}, 0, a\right)$ is equal to the multiplicity of the function $f_{j}^{(a)}(z)=f_{j}\left(z^{a}\right)$.

We also mention that (35) gives a lower bound for the Milnor number $\mu(F, 0)$ of a singular point 0 of a holomorphic function $F$ (i.e. for the multiplicity of the isolated zero of the mapping $f=\operatorname{grad} F$ at 0$)$ in terms of the indices $I(F, 0, a)$ (see $(7))$ of $F$. Since $I\left(\partial F / \partial z_{k}, 0, a\right) \geq I(F, 0, a)-a_{k}$, we have

$$
\mu(F, 0) \geq \prod_{1 \leq k \leq n}\left(\frac{I(F, 0, a)}{a_{k}}-1\right) .
$$

Finally, it follows from (7) that the set $\overline{\mathbb{R}_{+}^{n} \backslash \Theta_{u, 0}}$ is the Newton polyhedron for the system $\left(f_{1}, \ldots, f_{n}\right)$ at 0 (see Introduction). Therefore, $n ! V\left(\Theta_{u, 0}\right)$ is the Newton number of $\left(f_{1}, \ldots, f_{n}\right)$ at 0 , and (36) becomes the bound for $m_{f}$ due to A. G. Kouchnirenko (see [1], Th. 22.8). So, for any plurisubharmonic function $u$, we will call the value $N(u, x)$ the Newton number of $u$ at $x$. 
5. Indicators as logarithmic tangents. Let $u \in \operatorname{PSH}(\Omega, 0), u(0)=$ $-\infty$. We will consider the following problem: under what conditions on $u$, does its residual measure equal its Newton number?

Of course, the relation

$$
\lim _{z \rightarrow 0} \frac{u(z)}{\Psi_{u, 0}(z)}=1
$$

is sufficient, but it seems to be too restrictive. On the other hand, as the example $u(z)=\log \left(\left|z_{1}+z_{2}\right|^{2}+\left|z_{2}\right|^{4}\right)$ shows, the condition

$$
\lim _{\lambda \rightarrow 0} \frac{u(\lambda z)}{\Psi_{u, 0}(\lambda z)}=1 \quad \forall z \in \mathbb{C}^{n} \backslash\{0\}
$$

does not guarantee the equality $\tau(u, 0)=N(u, 0)$.

To weaken (37) we first give another description for the local indicators. In [6], a compact family of plurisubharmonic functions

$$
u_{r}(z)=u(r z)-\sup \{u(y):|y|<r\}, \quad r>0,
$$

was considered and the limit sets, as $r \rightarrow 0$, of such families were described. In particular, the limit set need not consist of a single function, so a plurisubharmonic function can have several (and thus infinitely many) tangents. Here we consider another family generated by a plurisubharmonic function $u$.

Given $m \in \mathbb{N}$ and $z \in \mathbb{C}^{n}$, write $z^{m}=\left(z_{1}^{m}, \ldots, z_{n}^{m}\right)$ and set

$$
T_{m} u(z)=m^{-1} u\left(z^{m}\right) .
$$

Clearly, $T_{m} u \in \operatorname{PSH}(\Omega \cap D)$ and $T_{m} u \in \operatorname{PSH}_{-}\left(\bar{D}_{r}\right)$ for any $r \in \mathbb{R}_{+}^{n} \cap D^{*}$ (i.e. $\left.0<r_{k}<1\right)$ for all $m \geq m_{0}(r)$.

Proposition 8. The family $\left\{T_{m} u\right\}_{m \geq m_{0}(r)}$ is compact in $L_{\mathrm{loc}}^{1}\left(D_{r}\right)$.

Proof. Let $M(v, \varrho)$ denote the mean value of a function $v$ over the set $\left\{z:\left|z_{k}\right|=\varrho_{k}, 1 \leq k \leq n\right\}, 0<\varrho_{k} \leq r_{k}$. Then $M\left(T_{m} u, \varrho\right)=m^{-1} M\left(u, \varrho^{m}\right)$. The relation

$$
m^{-1} M\left(u, \varrho^{m}\right) \nearrow \Psi_{u, 0}(\varrho) \quad \text { as } m \rightarrow \infty
$$

implies $M\left(T_{m} u, \varrho\right) \geq M\left(T_{m_{0}} u, \varrho\right)$. Since $T_{m} u \leq 0$ in $D_{r}$, this proves the compactness.

TheOREM 8. (a) $T_{m} u \rightarrow \Psi_{u, 0}$ in $L_{\mathrm{loc}}^{1}(D)$;

(b) if $u \in \operatorname{PSH}(\Omega, 0)$ then $\left(d d^{c} T_{m} u\right)^{n} \rightarrow \tau(u, 0) \delta(0)$.

Proof. Let $g$ be a limit point of the sequence $T_{m} u$, that is, $T_{m_{s}} u \rightarrow g$ as $s \rightarrow \infty$ for some sequence $m_{s}$. For the function $v(z)=\sup \left\{u(y):\left|y_{k}\right| \leq\right.$ $\left.\left|z_{k}\right|, 1 \leq k \leq n\right\}$ and any $r \in \mathbb{R}_{+}^{n} \cap D^{*}$ we have, by (33),

$$
T_{m} u(z) \leq\left(T_{m} v\right)(z) \leq \Psi_{u, 0}\left(r^{-1} \cdot z\right)
$$


and thus

$$
g(z) \leq \Psi_{u, 0}(z) \quad \forall z \in D .
$$

On the other hand, the convergence of $T_{m_{s}} u$ to $g$ in $L^{1}$ implies $M\left(T_{m_{s}} u, r\right)$ $\rightarrow M(g, r)$ ([3], Prop. 4.1.10). By (38), $M\left(T_{m_{s}} u, r\right) \rightarrow \Psi_{u, 0}(r)$, so $M(g, r)=$ $\Psi_{u, 0}(r)$ for every $r \in \mathbb{R}_{+}^{n} \cap D^{*}$. Comparison with (39) gives us $g \equiv \Psi_{u, 0}$, and the statement (a) follows.

To prove (b) we observe that for each $\alpha \in(0,1)$,

$$
\int_{\alpha D}\left(d d^{c} T_{m} u\right)^{n}=\int_{\alpha^{m} D}\left(d d^{c} u\right)^{n} \rightarrow \tau(u, 0)
$$

as $m \rightarrow \infty$, and for $0<\alpha<\beta<1$,

$$
\lim _{m \rightarrow \infty} \int_{\beta D \backslash \alpha D}\left(d d^{c} T_{m} u\right)^{n}=\lim _{m \rightarrow \infty}\left[\int_{\beta^{m} D}\left(d d^{c} u\right)^{n}-\int_{\alpha^{m} D}\left(d d^{c} u\right)^{n}\right]=0 .
$$

The theorem is proved.

So, Theorem 8 shows us that $\tau(u, 0)=N(u, 0)$ if and only if $\left(d d^{c} T_{m} u\right)^{n}$ $\rightarrow\left(d d^{c} \Psi_{u, 0}\right)^{n}$. Now we are going to find conditions for this convergence.

Recall the definition of the inner $\mathcal{C}_{n-1}$-capacity introduced in [17]: for any Borel subset $E$ of a domain $\omega$,

$$
\mathcal{C}_{n-1}(E, \omega)=\sup \left\{\int_{E}\left(d d^{c} v\right)^{n-1} \wedge \beta_{1}: v \in \operatorname{PSH}(\omega), 0<v<1\right\} .
$$

It was shown in [17] that convergence of uniformly bounded plurisubharmonic functions $v_{j}$ to $v$ in $\mathcal{C}_{n-1}$-capacity implies $\left(d d^{c} v_{j}\right)^{n} \rightarrow\left(d d^{c} v\right)^{n}$. In our situation, neither $T_{m} u$ nor $\Psi_{u, 0}$ are bounded, so we will modify the construction from [17].

Set

$$
E(u, m, \delta)=\left\{z \in D \backslash\{0\}: \frac{T_{m} u(z)}{\Psi_{u, 0}(z)}>1+\delta\right\}, \quad m \in \mathbb{N}, \delta>0 .
$$

Theorem 9. Let $u \in \operatorname{PSH}(\Omega, 0), \varrho \in(0,1 / 4), N>0$, and a sequence $m_{s} \in \mathbb{N}$ be such that

1) $u(z)>-N m_{s}$ on a neighbourhood of the sphere $\partial B_{\varrho^{m_{s}}}$, for each $s$;

2) $\lim _{s \rightarrow \infty} \mathcal{C}_{n-1}\left(B_{\varrho} \cap E\left(u, m_{s}, \delta\right), D\right)=0$ for all $\delta>0$.

Then $\left(d d^{c} T_{m} u\right)^{n} \rightarrow\left(d d^{c} \Psi_{u, 0}\right)^{n}$ on $D$.

Proof. Without loss of generality we can take $u \in \mathrm{PSH}_{-}(D, 0)$. Consider the functions $v_{s}(z)=\max \left\{T_{m_{s}} u(z),-N\right\}$ and $v=\max \left\{\Psi_{u, 0}(z),-N\right\}$. We have $v_{s}=T_{m_{s}} u$ and $v=\Psi_{u, 0}$ on a neighbourhood of $\partial B_{\varrho}, v_{s}=v=-N$ on a neighbourhood of $0, v_{s} \leq v$ on $B_{\varrho}$, and $v_{s} \geq(1+\delta) v$ on $B_{\varrho} \backslash E\left(u, m_{s}, \delta\right)$. 
We will prove that

$$
\left(d d^{c} v_{s}\right)^{k} \wedge\left(d d^{c} v\right)^{l} \rightarrow\left(d d^{c} v\right)^{k+l}
$$

for $k=1, \ldots, n, l=0, \ldots, n-k$. This will give us the statement of the theorem. Indeed, by Theorem 8,

$$
\int_{B_{\varrho}}\left(d d^{c} v_{s}\right)^{n}=\int_{B_{\varrho}}\left(d d^{c} T_{m_{s}} u\right)^{n} \rightarrow \tau(u, 0)
$$

while

$$
\int_{B_{\varrho}}\left(d d^{c} v\right)^{n}=\int_{B_{\varrho}}\left(d d^{c} \Psi_{u, 0}\right)^{n}=N(u, 0),
$$

and (40) with $k=n$ proves the coincidence of the right-hand sides of these relations and thus the convergence of $\left(d d^{c} T_{m} u\right)^{n}$ to $\left(d d^{c} \Psi_{u, 0}\right)^{n}$.

We prove (40) by induction on $k$. Let $k=1,0 \leq l \leq n-1, \delta>0$. For any test form $\phi \in \mathcal{D}_{n-l-1, n-l-1}\left(B_{\varrho}\right)$,

$$
\begin{aligned}
& \left|\int d d^{c} v_{s} \wedge\left(d d^{c} v\right)^{l} \wedge \phi-\int\left(d d^{c} v\right)^{l+1} \wedge \phi\right| \\
& \quad=\left|\int\left(v-v_{s}\right)\left(d d^{c} v\right)^{l} \wedge d d^{c} \phi\right| \leq C_{\phi} \int_{B_{\varrho}}\left(v-v_{s}\right)\left(d d^{c} v\right)^{l} \wedge \beta_{n-l} \\
& \quad=C_{\phi}\left[\int_{B_{\varrho} \backslash E_{s, \delta}}+\int_{B_{\varrho} \cap E_{s, \delta}}\right]\left(v-v_{s}\right)\left(d d^{c} v\right)^{l} \wedge \beta_{n-l}=C_{\phi}\left[I_{1}(s, \delta)+I_{2}(s, \delta)\right],
\end{aligned}
$$

where, for brevity, $E_{s, \delta}=E\left(u, m_{s}, \delta\right)$.

We have

$$
I_{1}(s, \delta) \leq \delta \int_{B_{\varrho}}|v|\left(d d^{c} v\right)^{l} \wedge \beta_{n-l} \leq C \delta
$$

with a constant $C$ independent of $s$, and

$$
\begin{aligned}
I_{2}(s, \delta) & \leq N \int_{B_{\varrho} \cap E_{s, \delta}}\left(d d^{c} v\right)^{l} \wedge \beta_{n-l} \\
& \leq C(N, \varrho, l) \cdot \mathcal{C}_{n-1}\left(B_{\varrho} \cap E_{s, \delta}, D\right) \rightarrow 0 .
\end{aligned}
$$

Since $\delta>0$ is arbitrary, this proves (40) for $k=1$.

Suppose that we have (40) for $k=j$ and $0 \leq l \leq n-j$. For $\phi \in$ $\mathcal{D}_{n-l-j, n-l}\left(B_{\varrho}\right)$,

$$
\begin{aligned}
& \int\left(d d^{c} v_{s}\right)^{j+1} \wedge\left(d d^{c} v\right)^{l} \wedge \phi \\
&+\int\left(d d^{c} v_{s}\right)^{j} \wedge\left(d d^{c} v\right)^{l+1} \wedge \phi \\
&+\int\left[\left(d d^{c} v_{s}\right)^{j+1} \wedge\left(d d^{c} v\right)^{l}-\left(d d^{c} v_{s}\right)^{j} \wedge\left(d d^{c} v\right)^{l+1}\right] \wedge \phi
\end{aligned}
$$

The first integral on the right-hand side converges to $\int\left(d d^{c} v\right)^{l+j+1} \wedge \phi$ by the induction assumption. The second integral can be estimated similarly 
to the case $k=1$ :

$$
\begin{aligned}
\mid \int\left[\left(d d^{c} v_{s}\right)^{j+1}\right. & \left.\wedge\left(d d^{c} v\right)^{l}-\left(d d^{c} v_{s}\right)^{j} \wedge\left(d d^{c} v\right)^{l+1}\right] \wedge \phi \mid \\
\leq & C_{\phi}\left[\int_{B_{\varrho} \backslash E_{s, \delta}}+\int_{B_{\varrho} \cap E_{s, \delta}}\right]\left(v-v_{s}\right)\left(d d^{c} v_{s}\right)^{j}\left(d d^{c} v\right)^{l} \wedge \beta_{n-j-l} \\
& =C_{\phi}\left[I_{3}(s, \delta)+I_{4}(s, \delta)\right] .
\end{aligned}
$$

Since $\left(d d^{c} v_{s}\right)^{j} \wedge\left(d d^{c} v\right)^{l} \rightarrow\left(d d^{c} v\right)^{j+l}$, we have

$$
\int\left(d d^{c} v_{s}\right)^{j}\left(d d^{c} v\right)^{l} \wedge \beta_{n-j-l} \leq C \quad \forall s
$$

and

$$
I_{3}(s, \delta) \leq \delta \int_{B_{\varrho}}|v|\left(d d^{c} v_{s}\right)^{j}\left(d d^{c} v\right)^{l} \wedge \beta_{n-j-l} \leq C N \delta
$$

Similarly,

$$
\begin{aligned}
I_{4}(s, \delta) & \leq N \int_{B_{\varrho} \cap E_{s, \delta}}\left(d d^{c} v_{s}\right)^{j}\left(d d^{c} v\right)^{l} \wedge \beta_{n-j-l} \\
& \leq C(N, \varrho, j, l) \cdot \mathcal{C}_{n-1}\left(B_{\varrho} \cap E_{s, \delta}, D\right) \rightarrow 0,
\end{aligned}
$$

and (40) is proved.

\section{References}

[1] L. A. A ̌̀zenberg and Yu. P. Yuzhakov, Integral Representations and Residues in Multidimensional Complex Analysis, Nauka, Novosibirsk, 1979 (in Russian); English transl.: AMS, Providence, RI, 1983.

[2] J.-P. Demailly, Monge-Ampère operators, Lelong numbers and intersection theory, in: Complex Analysis and Geometry, V. Ancona and A. Silva (eds.), Plenum Press, New York, 1993, 115-193.

[3] L. Hörmander, Notions of Convexity, Progr. Math. 127, Birkhäuser, 1994.

[4] C. O. Kiselman, Densité des fonctions plurisousharmoniques, Bull. Soc. Math. France 107 (1979), 295-304.

[5] —, Un nombre de Lelong raffiné, in: Séminaire d'Analyse Complexe et Géométrie 1985-87, Fac. Sci. Monastir, 1987, 61-70.

[6] - , Tangents of plurisubharmonic functions, in: International Symposium in Memory of Hua Loo Keng, Vol. II, Science Press and Springer, 1991, 157-167.

[7] - Attenuating the singularities of plurisubharmonic functions, Ann. Polon. Math. 60 (1994), 173-197.

[8] M. Klimek, Pluripotential Theory, Oxford Univ. Press, London, 1991.

[9] A. G. Kouchnirenko, Newton polyhedron and the number of solutions of a system of $k$ equations with $k$ indeterminates, Uspekhi Mat. Nauk 30 (1975), no. 2, 266-267 (in Russian).

[10] -, Polyèdres de Newton et nombres de Milnor, Invent. Math. 32 (1976), 1-31.

[11] S. Lang, Fundamentals of Diophantine Geometry, Springer, New York, 1983. 
[12] P. Lelong, Plurisubharmonic Functions and Positive Differential Forms, Gordon and Breach, New York, and Dunod, Paris, 1969.

[13] —, Remarks on pointwise multiplicities, Linear Topol. Spaces Complex Anal. 3 (1997), 112-119.

[14] P. Lelong and L. Gruman, Entire Functions of Several Complex Variables, Springer, Berlin, 1986.

[15] P. Lelong and A. Rashkovskii, Local indicators for plurisubharmonic functions, J. Math. Pures Appl. 78 (1999), 233-247.

[16] J. Rauch and B. A. Taylor, The Dirichlet problem for the multidimensional Monge-Ampère equation, Rocky Mountain J. Math. 7 (1977), 345-364.

[17] Y. Xing, Continuity of the complex Monge-Ampère operator, Proc. Amer. Math. Soc. 124 (1996), 457-467.

Mathematical Division

Institute for Low Temperature Physics

47 Lenin Ave.

Kharkov 310164, Ukraine

E-mail: rashkovskii@ilt.kharkov.ua

Reçu par la Rédaction le 20.7.1999

Révisé le 4.4.2000 